\title{
Interdisciplinary Science in Support of Environmental Health along the United States-Mexico Border
}

\author{
Using Map Tools to Understand Linkages between the Physical Environment and Human Health
}

The diverse, fragile ecosystems of the borderlands have been pushed beyond sustainable levels due to rapid population growth and land-use changes. Water shortages and pollution, poor air quality, increased soil salinities, residual pesticides, and heavy metal contaminants are some of the many stressors that are degrading the quality of life in the borderlands. The relationship between human health and environmental quality challenges public officials, medical professionals, and resource managers on both sides of the border in their efforts to provide for and maintain healthy communities. To help understand the relationship between environmental and human health, the U.S. Geological Survey's (USGS) Border Environmental Health Initiative (BEHI) created an Internet Map Service (IMS) with binational georeferenced data (fig. 1). The goal is to have seamless integration of borderwide datasets at regional and local scales that can lend understanding of the linkages between the condition of the physical environment and public health issues.

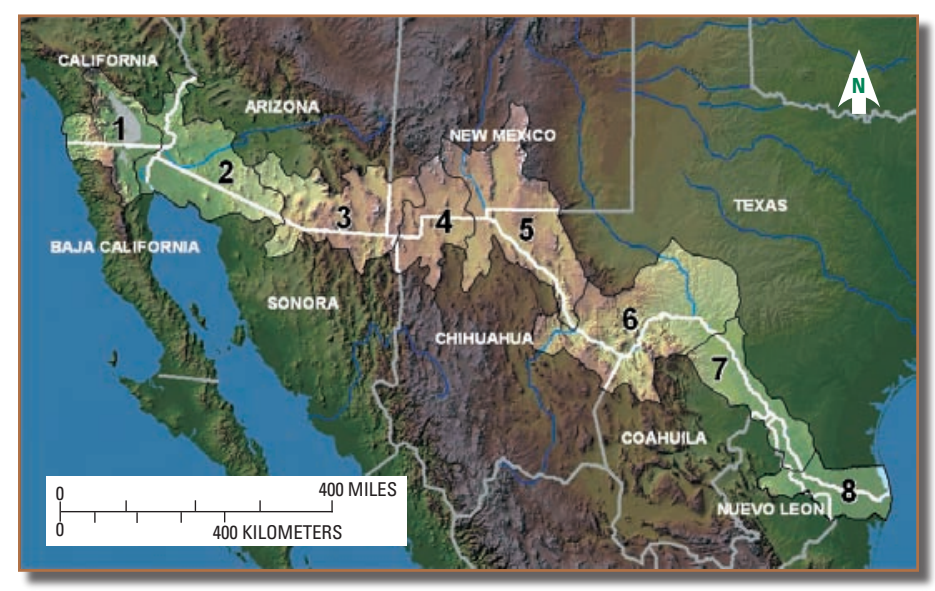

Figure 1. The USGS Border Environmental Health Initiative (BEHI) encompasses the entire United States-Mexico border region, an area defined as 161,000 square miles; it is partitioned into eight subregions based on watersheds as delineated by Woodward and Durall (1996).

Find the Border Environmental Health Initiative Internet Map Service at

http://borderhealth.cr.usgs.gov/

\section{Collaborative Partnerships}

An interdisciplinary USGS team identifies biologic, geologic, hydrologic, environmental, public health, and demographic datasets for incorporation into the binational IMS. This team works in collaboration with other Federal, State, and local agencies, including non-governmental entities and universities in both the United States and Mexico. Project success and the reliability of the binational scientific databases are dependent on these mutually beneficial partnerships.

Examples of cooperative partnerships that utilize our scientific data and research include:

Environmental Protection Agency/Secretaría de Medio Ambiente y Recursos Naturales

The Border 2012 Environmental Health Indicators Working Group focuses on developing metrics to measure changes in human health as a result of improvements to sanitation, air and water quality in the border region. The USGS is cooperating with this group to develop a binational water quality geodatabase for the region and to use geospatial data for reporting environmental activities.

\section{Natural Heritage Institute, University of Texas, and Instituto Mexicano de Tecnología del Agua \\ Through the Rio Grande Physical Assessment Project, the USGS is collaborating with United States and Mexican part- ners to build a water resources database as the foundation of an advanced hydrologic planning model of the entire Rio Grande basin. The USGS is verifying binational land-use and surface- and ground-water data, and validating model results.}

\section{Southwest Consortium of Environmental Resources Program (SCERP)}

The USGS is partnering with SCERP to further our respective missions and to enhance interaction and cooperation on border research and policy programs. The first joint activity was to co-host a binational GIS summit in April 2006.

\section{Housing and Urban Development 'Colonia' Database \\ This project is providing the necessary baseline data to identify the need for Federal aid to improve the living conditions for residents of the many unincorporated settle- ments, known as 'colonias,' found along the border. In conjunction with partners, the USGS is providing geographic and demographic data to facilitate prioritization of community development and infrastructure investment.}




\section{Data Assimilation and Integration}

USGS scientists strive to preserve the accuracy and associated attributes of the data during production of the IMS. Rigorous efforts have been made to seamlessly integrate United States and Mexican geospatial datasets along common themes to analyze environmental issues relative to human health. The procedures are documented in Federal Geographic Data Committeecompliant metadata files and in procedural papers that define the differences in scale, scope, definition of terms, and methods of data collection between the two countries.

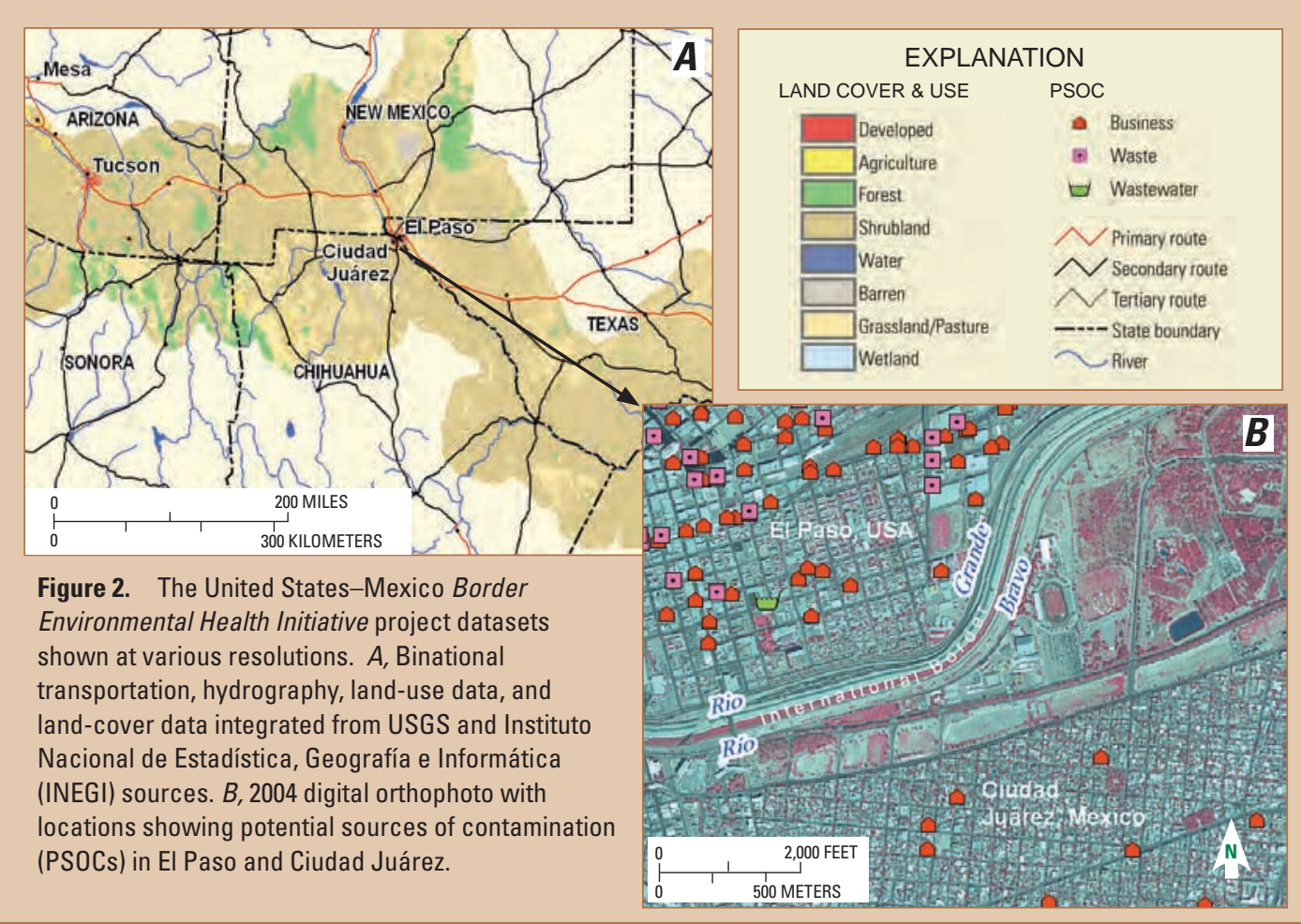

\section{Accomplishments to Date}

Methods for building binational geospatial data and constructing integrated databases were developed during the BEHI pilot phase for the Lower Rio Grande Valley (Subregion 8), which extends from Falcon Reservoir to the lower part of the Laguna Madre, Texas. The IMS for this data-rich area includes remotely sensed imagery, anthropogenic, and environmental datasets at a large scale $(1: 24,000)$. A similar suite of datasets at equivalent scales are being integrated for all subregions beginning with Subregion 7, which extends along the Rio Grande from Amistad Reservoir to Falcon Reservoir.

Applying similar methodology borderwide, regional datasets at medium- (1:250,000 scale) and low-resolution (1:2M scale) have been integrated to form the basic geospatial framework and are displayed in the IMS. These borderwide datasets provide the platform upon which the comprehensive and subregion-specific datasets will subsequently be overlaid, and also assure compatibility at temporal and spatial scales (fig. 2).

\section{Collaborative Opportunities}

An anticipated outcome of this project is an increased opportunity to collaborate with scientific researchers in the public health, natural resources, and environmental protection fields to apply the BEHI datasets and the IMS to address specific public and environmental health issues. The vision of the USGS is that such collaborations could ultimately expand the breadth and depth of the datasets available and allow more sophisticated analysis of border health issues, ultimately leading to a healthier border environment.

Woodward, D., and Durall, R., 1996, United States-Mexico border area, as delineated by a shared-water resources perspective: U.S. Department of Interior United States-Mexico Border Field Coordinating Committee Fact Sheet, February 1996.

\section{Web Site Features}

Maps and Data - The IMS provides users with binational datasets and the tools to manipulate them over the Internet using a browser. The interface allows users to zoom to areas of interest and select a combination of layers appropriate to their focus. Users can select an initial view of the entire border region or a particular subregion, and use the tools available to customize their view.

Data Layers - Data layers are grouped under the major themes of anthropology, hydrology, transportation, biology, geology, imagery, elevation, land use, and infectious disease. The numerous data layers within these themes are detailed with a description, metadata, and minimum and maximum viewable extents. By means of the web mapping service protocols, users may incorporate available layers into their own geospatial analyses.

Static Map Library - This feature provides users with ready-made maps of common views and themes. The maps can be easily integrated into custom presentations.

Data Tables - Additional information about the specific data used in the layers is provided in tabular form.

\section{For more information, contact:}

Jim Stefanov-Water Resources jestefan@usgs.gov

Diana Papoulias-Biology dpapoulias@usgs.gov
Jean Parcher-Geography jwparcher@usgs.gov

Ric Page-Geology rpage@usgs.gov 